\title{
A COMBINATION OF FRIEDEL-CRAFTS AND LAWESSON REACTIONS TO 5-SUBSTITUTED 2,2'-BITHIOPHENES
}

\author{
M. Manuela M. Raposo*a and G. Kirschb \\ a Departamento de Química, Universidade do Minho, Campus de Gualtar \\ 4710-057 Braga, Portugal, e-mail mfox@quimica.uminho.pt \\ b Laboratoire d’Ingénierie Moléculaire et Biochimie Pharmacologique, \\ Université de Metz, Ile de Saulcy, F-57405 Metz Cedex, France, e-mail \\ Kirsch@sciences.univ-metz.fr
}

\begin{abstract}
Keto esters (2) derivatives of thiophene were obtained from hemisuccinic esters and transformed to the corresponding amides (4). Lawesson's treatment of $\mathbf{2}$ and $\mathbf{4}$ gave the corresponding bithiophenes (5) with alkoxy or amino substituents.
\end{abstract}

In the past few years interest has been focused on new organic materials with non-linear optical properties, ${ }^{1-3}$ and it was proved that, introduction of the thiophene nucleus enhance these properties. ${ }^{4-6}$ Thiophenes and oligothiophenes substituted by donor-acceptor groups have been extensively investigated. ${ }^{7-11}$ These compounds are often used as energy transfer and light-harvesting systems ${ }^{12-14}$ and for optical and electronic devices. ${ }^{6}, 10-11$ Unsymetrically substituted bithiophenes are usually synthesised by cross-coupling reactions; Stille, $, 8,15-18$ Suzuki, ${ }^{19}$ or others ${ }^{20-22}$ and by procedures involving thiophene ring formations. ${ }^{23-24}$ As part of our continuing interest in non-linear optical material ${ }^{25-26}$ we have synthesised several 5-substituted 2,2`-bithiophenes via a combination of Friedel-Crafts and Lawesson reactions.

Starting from succinic anhydride, we have prepared the acid chlorides (1) which on Friedel-Crafts reaction with thiophene afforded the $\gamma$-keto esters (2). Hydrolysis of 2 gave the $\gamma$-keto acid (3) (Scheme 1). 27-30

Transformation of 3 to amide (4) was made via two different synthetic routes (Scheme 2). The mixed anhydride was prepared and reacted with the amine in DMF (Method A) (Table 1). In these cases, low 
yields and a secondary product, the $\boldsymbol{\gamma}$-keto ester (2b) were always obtained. Direct amidation of the acid (3) with the amine was then run through DCC-BtOH catalysed reaction (Method B). No secondary products were detected and the yields were moderate to good (Table 1).<smiles>O=C1CCC(=O)O1</smiles><smiles>CCCC</smiles><smiles>[R]OC(=O)CCC(=O)Cl</smiles>

$$
1 \mathbf{a}-\mathbf{c}
$$<smiles>[R]C(=O)CC(=O)c1cccs1</smiles>

1a and $\mathbf{2 a} \mathrm{R}=\mathrm{OMe}$

$\mathbf{1 b}$ and $\mathbf{2 b} \mathrm{R}=\mathrm{OEt}$

$1 \mathrm{c}$ and $2 \mathrm{c} \mathrm{R}=\mathrm{OPr}-i$<smiles>O=C(O)CC(=O)c1cccs1</smiles>

Reagents and conditions: i, $\mathrm{RH}$, reflux; ii, $\mathrm{SOCl}_{2}, \mathrm{CH}_{2} \mathrm{Cl}_{2}$, reflux; iii, Thiophene, $\mathrm{SnCl}_{4}, \mathrm{CH}_{2} \mathrm{Cl}_{2} ; \mathrm{iv}, \mathrm{NaOH}, 95 \% \mathrm{EtOH}$, reflux.

Scheme 1

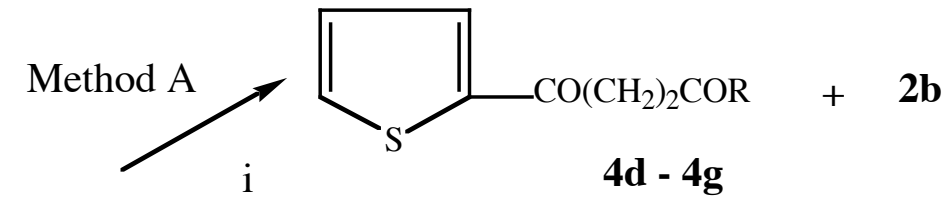

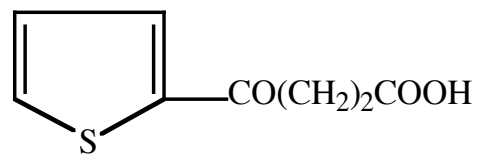

3

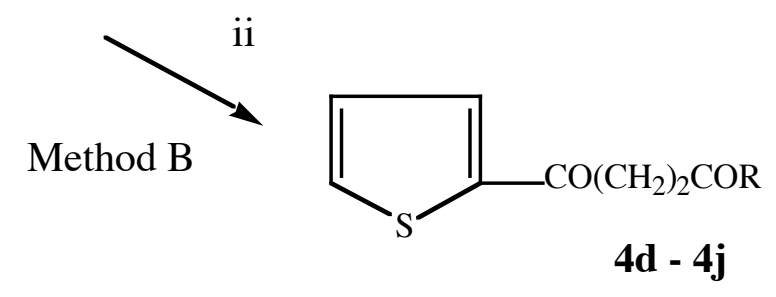

$$
\begin{aligned}
& \text { 4d } \mathrm{R}=\mathrm{NMe}_{2} \\
& \text { 4e } \mathrm{R}=\mathrm{NEt}_{2} \\
& \text { 4f } \mathrm{R}=\mathrm{N}[\mathrm{Pr}-i]_{2}
\end{aligned}
$$$$
4 \mathrm{~g} \quad \mathrm{R}=\mathrm{N}
$$$$
\text { 4h } \quad \mathrm{R}=\mathrm{N} \longrightarrow \square]_{2}
$$$$
\text { 4i } \mathrm{R}=\mathrm{HN}
$$

$4 \mathrm{j}=\mathrm{RN}$

Reagents and conditions: i, EtOCOCl, $\mathrm{NEt}_{3}, \mathrm{DMF}$, amine; ii, $\mathrm{DCC}, \mathrm{BtOH}, \mathrm{CH}_{2} \mathrm{Cl}_{2}$, amine.

\section{Scheme 2}


Table 1 Comparative yields of compounds (4) from $\gamma$-keto acid (3).

\begin{tabular}{|c|c|c|c|}
\hline Compound & $\mathbf{R}$ & Method A (\%) & Method B (\%) \\
\hline 4d & $\mathrm{NMe}_{2}$ & 15 & 70 \\
\hline $4 e$ & $\mathrm{NEt}_{2}$ & 40 & 67 \\
\hline $4 f$ & $\mathrm{~N}(\operatorname{Pr}-i)_{2}$ & 35 & 33 \\
\hline $4 g$ & & 50 & 84 \\
\hline $4 h$ & & ---- & 52 \\
\hline $4 \mathbf{i}$ & & ---- & 62 \\
\hline $\mathbf{4 j}$ & & ---- & 64 \\
\hline
\end{tabular}

Treatment of the esters (2a-c) and the amides (4d-i) with Lawesson's reagent (LR) afforded the corresponding alkoxy- and aminobithiophenes (5) (Scheme 3). The mechanism has been discussed by $\mathrm{T}$. Nisho ${ }^{31}$ and it has been shown that pyrroles may become by-products in the case of secondary amides, this may explain the low field of $\mathbf{5 i}$.

The yields of alkoxybithiophenes (5a-b) were improved on addition of a $20 \%$ excess of Lawesson's reagent (Table 2). 


\begin{tabular}{|c|c|c|}
\hline $\begin{array}{l}2 a-c \\
4 d-i\end{array}$ & & $5 \mathbf{a}-\mathbf{i}$ \\
\hline $2 \mathbf{a}$ & $\mathrm{R}=\mathrm{OMe}$ & $5 \mathbf{a}$ \\
\hline $2 \mathbf{b}$ & $\mathrm{R}=\mathrm{OEt}$ & $5 b$ \\
\hline $2 c$ & $\mathrm{R}=\mathrm{OPr}-i$ & $5 c$ \\
\hline 4d & $\mathrm{R}=\mathrm{NMe}_{2}$ & $5 d$ \\
\hline $4 e$ & $\mathrm{R}=\mathrm{NEt}_{2}$ & $5 e$ \\
\hline $\mathbf{4 f}$ & $\mathrm{R}=\mathrm{N}(\operatorname{Pr}-i)_{2}$ & $5 f$ \\
\hline $4 g$ & & $5 g$ \\
\hline $4 h$ & $\mathrm{R}=$ & $5 \mathrm{~h}$ \\
\hline $4 \mathbf{i}$ & $\mathrm{R}=\mathrm{HI}$ & $5 \mathbf{i}$ \\
\hline
\end{tabular}

Reagents and conditions: i, Lawesson's reagent, toluene, reflux.

\section{Scheme 3}

Table 2 Comparative yields of compounds (5a-c) from $\gamma$-keto esters (2)

\begin{tabular}{cccc}
\hline Compound & $\begin{array}{c}\text { Reaction time } \\
\text { (h) }\end{array}$ & $\begin{array}{c}\text { Yield (\%) with } \\
\text { equimolar amount of } \\
\text { LR }\end{array}$ & $\begin{array}{c}\text { Yield (\%) with } \\
\text { 20\% excess of LR }\end{array}$ \\
\hline 5a & 2 & 36 & 43 \\
5b & 1 & 31 & 49 \\
5c & 1 & 39 & ---- \\
\hline
\end{tabular}


In the case of the amides (4) no excess of Lawesson's reagent was used and the yields were moderate (Table 3). In fact the reaction runs faster than in the case of the esters.

Table 3 Yields of compounds (5d - i) from amides (4)

\begin{tabular}{|c|c|c|c|}
\hline Compound & $\mathbf{R}$ & Yield $(\%)$ & $\begin{array}{c}\text { Reaction time } \\
(\text { min) }\end{array}$ \\
\hline $5 d$ & $\mathrm{NMe}_{2}$ & 69 & 30 \\
\hline $5 e$ & $\mathrm{NEt}_{2}$ & 64 & 25 \\
\hline $5 f$ & $\mathrm{~N}(\operatorname{Pr}-i)_{2}$ & 53 & 35 \\
\hline $5 g$ & & 79 & 35 \\
\hline $5 \mathrm{~h}$ & & 63 & 60 \\
\hline $5 \mathbf{i}$ & & 14 & 15 \\
\hline
\end{tabular}

\section{EXPERIMENTAL}

${ }^{1} \mathrm{H}$ NMR spectra were obtained on a Varian Unity Plus Spectrometer at $300 \mathrm{MHz}$ and ${ }^{13} \mathrm{C}$ NMR spectra were determinated in the same instrument at 75.4 MHz. The solvent are quoted in parenthesis before the chemical shift values ( $\delta$ relative to internal $\mathrm{CDCl}_{3}$ ). Mp were determinated on a Gallenkamp apparatus and are uncorrected. IR spectra were recorded as Nujol mulls, on a Perkin Elmer 1600 FTIR Spectrophotometer, unless stated otherwise. UV spectra were recorded in ethanol on a Hitachi U-2000. EI MS were run on a Unicam GC-MS 120. Elemental analyses were made on a Leco CHNS-932. Column chromatography was performed on Merck silica gel 60 (Art 9385). Light petroleum refers to solvent boiling in the range $40-60{ }^{\circ} \mathrm{C}$.

\section{General procedure for the preparation of acid chlorides (1) .}

The acid chlorides $(70 \mathrm{mmol}$ ) were dissolved in $50 \mathrm{~mL}$ of dry dichloromethane and then thionyl chloride $(12.5 \mathrm{~g}, 105 \mathrm{mmol})$ was added and the mixture was heated at reflux for 4-4.5 h. Evaporation of the solvent 
under reduced pressure gave the crude acid chlorides (1) which were used without further purification in the Friedel-Crafts reactions.

3-Carbomethoxypropionyl chloride (1a) : colorless oil. Quantitative yield. ${ }^{1} \mathrm{H}$ NMR $\delta_{\mathrm{H}}\left(\mathrm{CDCl}_{3}\right) 2.80$ $\left(2 \mathrm{H}, \mathrm{t}, \underline{\mathrm{J}}=6.6 \mathrm{~Hz}, \underline{\mathrm{C}}_{2}\right), 3.20\left(2 \mathrm{H}, \mathrm{t}, \underline{\mathrm{J}}=6.6 \mathrm{~Hz}, \underline{\mathrm{C}}_{2}\right), 3.71\left(3 \mathrm{H}, \mathrm{s}, \mathrm{OC} \underline{\mathrm{H}}_{3}\right)$.

3-Carboethoxypropionyl chloride (1b) ${ }^{27}$ : colorless oil. Quantitative yield. ${ }^{1} \mathrm{H}$ NMR $\delta_{\mathrm{H}}\left(\mathrm{CDCl}_{3}\right) 1.25$ $\left(2 \mathrm{H}, \mathrm{t}, \underline{\mathrm{J}}=6.7 \mathrm{~Hz}, \mathrm{OCH}_{2} \mathrm{C}_{3}\right), 2.65\left(2 \mathrm{H}, \mathrm{t}, \underline{\mathrm{J}}=6.7 \mathrm{~Hz}, \mathrm{C}_{2}\right), 3.20\left(2 \mathrm{H}, \mathrm{t}, \underline{\mathrm{J}}=6.7 \mathrm{~Hz}, \underline{\mathrm{C}}_{2}\right), 4.15(2 \mathrm{H}, \mathrm{q}$ $\left.\underline{\mathrm{J}}=6.7 \mathrm{~Hz}, \mathrm{OCH}_{2} \mathrm{CH}_{3}\right)$.

3-Carboisopropoxypropionyl chloride (1c) : colorless oil. Quantitative yield. ${ }^{1} \mathrm{H} \mathrm{NMR} \delta_{\mathrm{H}}\left(\mathrm{CDCl}_{3}\right) 1.23$ $\left(6 \mathrm{H}, \mathrm{d}, \underline{\mathrm{J}}=6.3 \mathrm{~Hz}, \mathrm{OCH}\left(\mathrm{C}_{3}\right)_{2}\right), 2.63\left(2 \mathrm{H}, \mathrm{t}, \underline{\mathrm{J}}=6.3 \mathrm{~Hz}, \underline{\mathrm{C}}_{2}\right), 3.19\left(2 \mathrm{H}, \mathrm{t}, \underline{\mathrm{J}}=6.3 \mathrm{~Hz}, \mathrm{C}_{2}\right), 5.05(1 \mathrm{H}$, sep, $\left.\underline{\mathrm{J}}=6.3 \mathrm{~Hz}, \underline{\mathrm{CH}}\left(\mathrm{CH}_{3}\right)_{2}\right)$.

\section{General procedure for the Friedel-Crafts reaction.}

Acid chloride (1) (70 mmol) and thiophene $(5.88 \mathrm{~g}, 70 \mathrm{mmol})$ in dry dichloromethane $(200 \mathrm{~mL})$ were added dropwise to a stirred solution of stannic chloride $(20 \mathrm{~g}, 77 \mathrm{mmol})$ in dry dichloromethane $(200 \mathrm{~mL})$ at $0{ }^{\circ} \mathrm{C}$. After the addition, the mixture was stirred overnight at $\mathrm{rt}$ and then poured onto ice-water (1000 $\mathrm{mL}$ ), acidified with conc. $\mathrm{HCl}$ and stirred at $0{ }^{\circ} \mathrm{C}$ during $1 \mathrm{~h}$. This mixture was extracted with dichoromethane $(3 \times 50 \mathrm{~mL})$ and the combined organic extracts were washed with a solution of $20 \% \mathrm{NaOH}$ $(3 \times 50 \mathrm{~mL})$, water $(2 \times 200 \mathrm{~mL})$ and dried $\left(\mathrm{Na}_{2} \mathrm{SO}_{4}\right)$. Evaporation of the solvent under reduced pressure gave the crude $\gamma$-keto esters (2) as oil which were purified by "flash" chromatography on silica with increasing amounts of ether in light petroleum as eluent.

Methyl 4-(2-thienyl)-4-oxobutanoate (2a) ${ }^{28}$ : yellow oil. Yield 83\%. Anal. Calcd for $\mathrm{C}_{9} \mathrm{H}_{10} \mathrm{O}_{3} \mathrm{~S}: \mathrm{C}, 54.55$; H, 5.05; S, 16.18. Found: C, 54.42; H, 5.18; S, 16.40. ${ }^{1} \mathrm{H}$ NMR $\delta_{\mathrm{H}}\left(\mathrm{CDCl}_{3}\right) 2.77\left(2 \mathrm{H}, \mathrm{t}, \underline{\mathrm{J}}=6.6 \mathrm{~Hz}, \mathrm{C}_{2}\right)$, $3.27\left(2 \mathrm{H}, \mathrm{t}, \underline{\mathrm{J}}=6.6 \mathrm{~Hz}, \mathrm{CH}_{2}\right), 3.71\left(3 \mathrm{H}, \mathrm{s}, \mathrm{OCH}_{3}\right), 7.12-7.16\left(1 \mathrm{H}, \mathrm{m}, 4^{\prime}-\mathrm{H}\right), 7.65(1 \mathrm{H}, \mathrm{dd}, \underline{\mathrm{J}}=5.2$ and 1.3 Hz, 5'-H), $7.78\left(1 \mathrm{H}, \mathrm{dd}, \underline{\mathrm{J}}=3.9\right.$ and $\left.1.3 \mathrm{~Hz}, 3^{\prime}-\mathrm{H}\right)$. MS (EI, $\left.70 \mathrm{eV}\right): \mathrm{m} / \mathrm{z}(\%) 198\left(\mathrm{M}^{+}, 17\right), 167$ (18), 111 (100), 83 (13), 55 (20). IR $v_{\max }\left(\right.$ liquid film) $/ \mathrm{cm}^{-1} 1739,1666$.

Ethyl 4-(2-thienyl)-4-oxobutanoate (2b) ${ }^{29}$ : pale yellow oil. Yield 83\%. Anal. Calcd for $\mathrm{C}_{10} \mathrm{H}_{12} \mathrm{O}_{3} \mathrm{~S}: \mathrm{C}$, 56.60; H, 5.65; S, 15.11. Found: C, 56.58; H, 5.79; S, 15.47. ${ }^{1} \mathrm{H} \mathrm{NMR} \delta_{\mathrm{H}}\left(\mathrm{CDCl}_{3}\right) 1.27(3 \mathrm{H}, \mathrm{t}, \underline{\mathrm{J}}=6.7 \mathrm{~Hz}$, $\left.\mathrm{OCH}_{2} \mathrm{C}_{3}\right), 2.76\left(2 \mathrm{H}, \mathrm{t}, \underline{\mathrm{J}}=6.7 \mathrm{~Hz}, \mathrm{C}_{2}\right), 3.26\left(2 \mathrm{H}, \mathrm{t}, \underline{\mathrm{J}}=6.7 \mathrm{~Hz}, \underline{\mathrm{C}}_{2}\right), 4.18(2 \mathrm{H}, \mathrm{q}, \underline{\mathrm{J}}=6.7 \mathrm{~Hz}$, $\mathrm{OCH}_{2} \mathrm{CH}_{3}, 7.12-7.16\left(1 \mathrm{H}, \mathrm{m}, 4^{\prime}-\mathrm{H}\right), 7.65\left(1 \mathrm{H}, \mathrm{dd}, \underline{\mathrm{J}}=5.1\right.$ and $\left.1.2 \mathrm{~Hz}, 5^{\prime}-\mathrm{H}\right), 7.78(1 \mathrm{H}, \mathrm{dd}, \underline{\mathrm{J}}=4.0$ and 1.2 Hz, 3'-H). MS (EI, 70 eV): m/z (\%) $213\left(\mathrm{M}^{+}+1\right.$ 1, 12) $212\left(\mathrm{M}^{+}, 57\right), 167$ (5), 83 (100), 69 (23), 55 (19). IR $v_{\max }($ liquid film)/cm-1 1730, 1666.

Isopropyl 4-(2-thienyl)-4-oxobutanoate (2c): yellow oil. Yield 5\%. Anal. Calcd for $\mathrm{C}_{11} \mathrm{H}_{14} \mathrm{O}_{3} \mathrm{~S}: \mathrm{C}, 58.41$; H, 6.19; S, 14.18. Found: C, 58.41; H, 6.11; S, 14.22. ${ }^{1} \mathrm{H}$ NMR $\delta_{\mathrm{H}}\left(\mathrm{CDCl}_{3}\right) 1.25(6 \mathrm{H}, \mathrm{d}, \underline{\mathrm{J}}=6.3 \mathrm{~Hz}$, 
$\left.\mathrm{OCH}\left(\mathrm{C}_{3}\right)_{2}\right), 2.74\left(2 \mathrm{H}, \mathrm{t}, \underline{\mathrm{J}}=6.3 \mathrm{~Hz}, \underline{\mathrm{C}}_{2}\right), 3.25\left(2 \mathrm{H}, \mathrm{t}, \underline{\mathrm{J}}=6.3 \mathrm{~Hz}, \mathrm{C}_{2}\right), 5.04(1 \mathrm{H}, \mathrm{sep}, \underline{\mathrm{J}}=6.3 \mathrm{~Hz}$, $\left.\mathrm{C} \underline{\mathrm{H}}\left(\mathrm{CH}_{3}\right)_{2}\right) 7.12-7.16\left(1 \mathrm{H}, \mathrm{m}, 4^{\prime}-\mathrm{H}\right), 7.66\left(1 \mathrm{H}, \mathrm{dd}, \underline{\mathrm{J}}=5.4\right.$ and $\left.1.4 \mathrm{~Hz}, 5^{\prime}-\mathrm{H}\right), 7.78(1 \mathrm{H}, \mathrm{dd}, \underline{\mathrm{J}}=4.1$ and 1.4 Hz, 3'-H). MS (EI, 70 eV): m/z (\%) 226 (M+, 12), 184 (4), 167 (42), 139 (8), 111 (100), 83 (13), 55 (24). IR $v_{\max }\left(\right.$ liquid film) $/ \mathrm{cm}^{-1} 1728,1667$.

4-Oxo-(2-thienyl)butanoic acid (3) ${ }^{30}$ : Ester (2b) $(1.5 \mathrm{~g}, 7 \mathrm{mmol})$ was heated at reflux for $1 \mathrm{~h}$ in a solution of sodium hydroxide $(0.57 \mathrm{~g}, 14 \mathrm{mmol})$ in $95 \%$ ethanol $(50 \mathrm{~mL})$. The mixture was cooled and after evaporation was poured onto water and extracted with chloroform $(3 \times 50 \mathrm{~mL})$. Acidification of the aqueous phase with conc. $\mathrm{HCl}$ till $\mathrm{pH} 1-2$ gave 3 as a pale pink solid, mp 119-120.5 ${ }^{\circ} \mathrm{C}$ [lit., 30 119-120 $\left.{ }^{\circ} \mathrm{C}\right]$. Yield (1 g, 78\%).

\section{General procedure for the synthesis of amides (4d-g); Method A: EtOCOCl / NEt 3 / DMF.}

Triethylamine $(4.48 \mathrm{~mL}, 32.4 \mathrm{mmol})$ and ethyl chloroformate $(3.12 \mathrm{~mL}, 32.4 \mathrm{mmol})$ were added dropwise to a stirred solution of the acid (3) $(2.0 \mathrm{~g}, 10.8 \mathrm{mmol})$ in dry dimethylformamide $(5 \mathrm{~mL})$ at $-5^{\circ} \mathrm{C}$. After $15 \mathrm{~min}$, the amine $(10.8 \mathrm{mmol})$ was added and the mixture was stirred overnight at $\mathrm{rt}$. Triethylamine hydrochloride was filtered off and washed with dimethylformamide. Evaporation of the solvent under reduced pressure gave the mixture of the amides (4d-g) and the $\gamma$-keto ester (2b) as oils which were isolated by "flash" chromatography on silica with increasing amounts of ether in light petroleum as eluent.

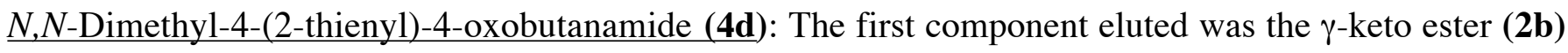
as a pale yellow oil. Yield $48 \%$. The second component eluted was the amide (4d) as a beige solid $\mathrm{mp}$ 96.2-97.7 ${ }^{\circ} \mathrm{C}$ (ether). Yield 15\%. Anal. Calcd for $\mathrm{C}_{10} \mathrm{H}_{13} \mathrm{NO}_{2} \mathrm{~S}: \mathrm{C}, 56.88 ; \mathrm{H}, 6.16$; N, 6.63; S, 15.18. Found: C, 56.64; H, 6.12; N, 6.50; S, 15.14. ${ }^{1} \mathrm{H} \mathrm{NMR} \delta_{\mathrm{H}}\left(\mathrm{CDCl}_{3}\right) 2.78\left(2 \mathrm{H}, \mathrm{t}, \underline{\mathrm{J}}=6.7 \mathrm{~Hz}, \mathrm{CH}_{2}\right), 2.97(3 \mathrm{H}$, s, $\left.\mathrm{NC}_{3}\right), 3.09\left(3 \mathrm{H}, \mathrm{s}, \mathrm{NC}_{3}\right), 3.31\left(2 \mathrm{H}, \mathrm{t}, \underline{\mathrm{J}}=6.7 \mathrm{~Hz}, \mathrm{CH}_{2}\right), 7.14\left(1 \mathrm{H}, \mathrm{m}, 4^{\prime}-\mathrm{H}\right), 7.63(1 \mathrm{H}, \mathrm{br} \mathrm{d}, \underline{\mathrm{J}}=5.2$ $\left.\mathrm{Hz}, 5^{\prime}-\mathrm{H}\right), 7.82$ (1H, br d, J = 3.7 Hz, 3'-H). MS (EI, $\left.70 \mathrm{eV}\right): \mathrm{m} / \mathrm{z}(\%) 211\left(\mathrm{M}^{+}, 76\right), 167$ (68), 139 (25), 111 (100), 100 (58), 83 (20), 72 (67), 69 (18), 55 (28). IR $v_{\max } / \mathrm{cm}^{-1} 1641$.

$N, N$-Diethyl-4-(2-thienyl)-4-oxobutanamide (4e): The first component eluted was the $\gamma$-keto ester (2b) as a pale yellow oil. Yield 23\%. The second component eluted was the amide (4e) as a beige solid, mp 62.5-65 ${ }^{\circ} \mathrm{C}$ (ether). Yield 40\%. Anal. Calcd for $\mathrm{C}_{12} \mathrm{H}_{17} \mathrm{NO}_{2} \mathrm{~S}: \mathrm{C}, 60.23 ; \mathrm{H}, 7.11 ; \mathrm{N}, 5.86 ; \mathrm{S}, 13.41$. Found: C, 60.44; H, 7.07; N, 5.80; S, 13.34. ${ }^{1} \mathrm{H} \mathrm{NMR}_{\mathrm{H}}\left(\mathrm{CDCl}_{3}\right) 1.11\left(3 \mathrm{H}, \mathrm{t}, \underline{\mathrm{J}}=7.5 \mathrm{~Hz}, \mathrm{CH}_{2} \mathrm{CH}_{3}\right), 1.23$ $\left(3 \mathrm{H}, \mathrm{t}, \underline{\mathrm{J}}=7.5 \mathrm{~Hz}, \mathrm{CH}_{2} \underline{\mathrm{C}}_{3}\right), 2.77\left(2 \mathrm{H}, \mathrm{t}, \underline{\mathrm{J}}=7.5 \mathrm{~Hz}, \underline{\mathrm{C}}_{2}\right), 3.31\left(2 \mathrm{H}, \mathrm{t}, \underline{\mathrm{J}}=7.5 \mathrm{~Hz}, \mathrm{C}_{2}\right), 3.40(4 \mathrm{H}, \mathrm{m}$, $\left.2 \mathrm{xC}_{2} \mathrm{CH}_{3}\right), 7.13\left(1 \mathrm{H}, \mathrm{m}, 4^{\prime}-\mathrm{H}\right), 7.62\left(1 \mathrm{H}, \mathrm{dd}, \underline{\mathrm{J}}=5.3\right.$ and $\left.1.2 \mathrm{~Hz}, 5^{\prime}-\mathrm{H}\right), 7.81(1 \mathrm{H}, \mathrm{dd}, \underline{\mathrm{J}}=4.1$ and 1.2 $\left.\mathrm{Hz}, 3^{\prime}-\mathrm{H}\right)$. MS (EI, $\left.70 \mathrm{eV}\right): \mathrm{m} / \mathrm{z}$ (\%) 239 (M+, 17), 211 (2), 167 (51), 140 (4), 128 (20), 111 (25), 100 (7), 72 (100), 58 (30). IR $v_{\max } / \mathrm{cm}^{-1} 1632$.

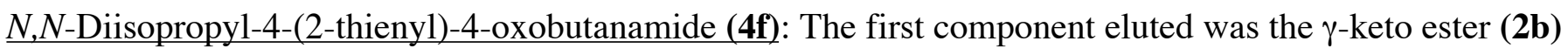
as a pale yellow oil. Yield 23\%. The second component eluted was the amide (4f) as a beige solid, $\mathrm{mp}$ 69-70.5 ${ }^{\circ} \mathrm{C}$ (ether). Yield 35\%. Anal. Calcd for $\mathrm{C}_{14} \mathrm{H}_{21} \mathrm{NO}_{2} \mathrm{~S}: \mathrm{C}, 62.93$; H, 7.86; N, 5.24; S, 12.0. Found: 
$\mathrm{C}, 62.76 ; \mathrm{H}, 8.00 ; \mathrm{N}, 5.27 ; \mathrm{S}, 12.27 .{ }^{1} \mathrm{H}$ NMR $\delta_{\mathrm{H}}\left(\mathrm{CDCl}_{3}\right) 1.25\left(6 \mathrm{H}, \mathrm{d}, \underline{\mathrm{J}}=7.2 \mathrm{~Hz}, \mathrm{CH}\left(\mathrm{CH}_{3}\right)_{2}\right), 1.38(6 \mathrm{H}$, d, $\left.\underline{\mathrm{J}}=7.2 \mathrm{~Hz}, \mathrm{CH}\left(\mathrm{C}_{3}\right)_{2}\right), 2.76\left(2 \mathrm{H}, \mathrm{t}, \underline{\mathrm{J}}=7.2 \mathrm{~Hz}, \underline{\mathrm{C}}_{2}\right), 3.31\left(2 \mathrm{H}, \mathrm{t}, \underline{\mathrm{J}}=7.2 \mathrm{~Hz}, \mathrm{C}_{2}\right), 3.90(1 \mathrm{H}, \mathrm{m}$, $\left.\mathrm{C} \underline{\mathrm{H}}\left(\mathrm{CH}_{3}\right)_{2}\right), 4.11\left(1 \mathrm{H}, \mathrm{m}, \mathrm{C} \underline{\mathrm{H}}\left(\mathrm{CH}_{3}\right)_{2}\right), 7.15-7.17\left(1 \mathrm{H}, \mathrm{m}, 4^{\prime}-\mathrm{H}\right), 7.62\left(1 \mathrm{H}, \mathrm{dd}, \underline{\mathrm{J}}=5.1\right.$ and $\left.1.2 \mathrm{~Hz}, 5^{\prime}-\mathrm{H}\right)$, $7.81\left(1 \mathrm{H}, \mathrm{dd}, \underline{\mathrm{J}}=3.9\right.$ and $\left.1.2 \mathrm{~Hz}, 3^{\prime}-\mathrm{H}\right)$. MS (EI, $\left.70 \mathrm{eV}\right): \mathrm{m} / \mathrm{z}(\%) 267\left(\mathrm{M}^{+}, 2\right), 167$ (20), 111 (31), 57 (100). IR $v_{\max } / \mathrm{cm}^{-1} 1636$.

Piperidino-4-(2-thienyl)-4-oxobutanamide (4g): The first component eluted was the $\gamma$-keto ester (2b) as a pale yellow oil. Yield $43 \%$. The second component eluted was the amide (4g) as a beige oil. Yield 50\%. Anal. Calcd for $\mathrm{C}_{13} \mathrm{H}_{17} \mathrm{NO}_{2} \mathrm{~S}: \mathrm{C}, 62.16 ; \mathrm{H}, 6.77 ; \mathrm{N}, 5.57 ; \mathrm{S}, 12.76$. Found: C, 62.25; H, 6.80; N, 5.55; S, 12.94. ${ }^{1} \mathrm{H}$ NMR $\delta_{\mathrm{H}}\left(\mathrm{CDCl}_{3}\right) 1.64\left(6 \mathrm{H}, \mathrm{m}, 3 \mathrm{xC}_{2}\right) 2.78\left(2 \mathrm{H}\right.$, br t $\left., \underline{\mathrm{J}}=6.4 \mathrm{~Hz}, \underline{\mathrm{C}}_{2}\right), 3.30(2 \mathrm{H}, \mathrm{br} \mathrm{t}, \underline{\mathrm{J}}=6.4$ $\left.\mathrm{Hz}, \mathrm{C}_{2}\right), 3.47\left(2 \mathrm{H}, \mathrm{t}, \underline{\mathrm{J}}=5.9 \mathrm{~Hz}, \mathrm{NC}_{2}\right), 3.56\left(2 \mathrm{H}, \mathrm{t}, \underline{\mathrm{J}}=5.9 \mathrm{~Hz}, \mathrm{NC}_{2}\right), 7.12-7.16\left(1 \mathrm{H}, \mathrm{m}, 4^{\prime}-\mathrm{H}\right), 7.63$ $\left(1 \mathrm{H}, \mathrm{dd}, \underline{\mathrm{J}}=5.3\right.$ and $\left.1.2 \mathrm{~Hz}, 5^{\prime}-\mathrm{H}\right), 7.82\left(1 \mathrm{H}, \mathrm{dd}, \underline{\mathrm{J}}=4.1\right.$ and $\left.1.2 \mathrm{~Hz}, 3^{\prime}-\mathrm{H}\right)$. MS $(\mathrm{EI}, 70 \mathrm{eV}): \mathrm{m} / \mathrm{z}(\%) 251$ $\left(\mathrm{M}^{+}, 12\right), 167$ (17), 139 (9), 125 (4), 111 (45), 97 (12), 84 (100), 56 (40). IR $v_{\max }$ (liquid film)/cm-1 1638.

\section{General procedure for the synthesis of amides (4d-j); Method B: DCC / BtOH / $\mathrm{CH}_{\mathbf{2}} \mathrm{Cl}_{\mathbf{2}}$.}

1,3-Dicyclohexylcarbodiimide (DCC) $(6.7 \mathrm{~g}, 32.6 \mathrm{mmol})$ and 1-hydroxybenzotriazole (BtOH) $(5 \mathrm{~g}, 32.6$ mmol) were added to a stirred solution of the acid (3) $(5.0 \mathrm{~g}, 27.2 \mathrm{mmol})$ in dichloromethane $(200 \mathrm{~mL})$ at $0{ }^{\circ} \mathrm{C}$. The mixture was stirred for $30 \mathrm{~min}$ at $\mathrm{rt}$ after which the amine $(27.2 \mathrm{mmol})$ was added and the mixture was stirred overnight. The by-product formed, dicyclohexylurea (DCU), was separated by filtration affording a pale brown solution. This organic solution was extracted with a solution of citric acid $(5 \%)(4 \times 100 \mathrm{~mL})$, a solution of sodium bicarbonate $(5 \%)(2 \times 100 \mathrm{~mL})$, dried $\left(\mathrm{MgSO}_{4}\right)$ and evaporated to give an oily brown residue. "Flash" chromatography on silica with increasing amounts of ether in light petroleum as eluent gave the pure amides $(\mathbf{4 d - j})$.

N,N-Dicyclohexyl-4-(2-thienyl)-4-oxobutanamide (4h): colorless solid mp 125-126 ${ }^{\circ} \mathrm{C}$ (ether). Yield $52 \%$. Anal. Calcd for $\mathrm{C}_{20} \mathrm{H}_{29} \mathrm{NO}_{2} \mathrm{~S}: \mathrm{C}, 69.17 ; \mathrm{H}, 8.35 ; \mathrm{N}, 4.03 ; \mathrm{S}, 9.23$. Found: C, 68.98; H, 8.32; N, 4.12; S, 9.33. ${ }^{1} \mathrm{H}$ NMR $\delta_{\mathrm{H}}\left(\mathrm{CDCl}_{3}\right) 1.00-2.00\left(20 \mathrm{H}, \mathrm{m}, 10 \mathrm{xC}_{2}\right) 2.40(1 \mathrm{H}, \mathrm{br} \mathrm{s}, \mathrm{C} \underline{\mathrm{H}}), 2.76(2 \mathrm{H}, \mathrm{t}, \underline{\mathrm{J}}=6.8$ $\left.\mathrm{Hz}, \mathrm{C}_{2}\right), 3.31\left(2 \mathrm{H}, \mathrm{t}, \underline{\mathrm{J}}=6.8 \mathrm{~Hz}, \underline{\mathrm{C}}_{2}\right), 3.60(1 \mathrm{H}, \mathrm{br} \mathrm{s}, \mathrm{C} \underline{\mathrm{H}}), 7.11-7.14\left(1 \mathrm{H}, \mathrm{m}, 4^{\prime}-\mathrm{H}\right), 7.61(1 \mathrm{H}, \mathrm{br} \mathrm{d}, \underline{\mathrm{J}}=$ $\left.4.9 \mathrm{~Hz}, 5^{\prime}-\mathrm{H}\right), 7.80\left(1 \mathrm{H}\right.$, br d, $\left.\underline{\mathrm{J}}=3.8 \mathrm{~Hz}, 3^{\prime}-\mathrm{H}\right)$. MS (EI, $\left.70 \mathrm{eV}\right): \mathrm{m} / \mathrm{z}(\%) 347\left(\mathrm{M}^{+}, 3\right), 290$ (2), 236 (7), 208 (2), 180 (21), 167 (100), 154 (9), 138 (19), 126 (5), 111 (26), 98 (19), 83 (6), 55 (15). IR $v_{\max }(\mathrm{KBr}) / \mathrm{cm}^{-1} 1665,1630$.

4-Methoxyphenyl-4-(2-thienyl)-4-oxobutanamide (4i): pale gray solid mp 139-140.5 ${ }^{\circ} \mathrm{C}$ (ethanol). Yield $62 \%$. Anal. Calcd for $\mathrm{C}_{15} \mathrm{H}_{15} \mathrm{NO}_{3} \mathrm{~S}: \mathrm{C}, 62.29 ; \mathrm{H}, 5.19 ; \mathrm{N}, 4.84 ; \mathrm{S}, 11.1$. Found: C, 62.1; H, 5.21; N, 4.71; $\mathrm{S}, 11.5 .{ }^{1} \mathrm{H}$ NMR $\delta_{\mathrm{H}}\left(\mathrm{CDCl}_{3}\right) 2.79\left(2 \mathrm{H}, \mathrm{t}, \underline{\mathrm{J}}=6.7 \mathrm{~Hz}, \mathrm{C}_{2}\right), 3.41\left(2 \mathrm{H}, \mathrm{t}, \underline{\mathrm{J}}=6.7 \mathrm{~Hz}, \mathrm{C}_{2}\right), 3.79(3 \mathrm{H}, \mathrm{s}$, $\left.\mathrm{OC}_{3}\right), 6.85\left(2 \mathrm{H}, \mathrm{d} \underline{\mathbf{J}}=8.9 \mathrm{~Hz}, 3^{\prime \prime}\right.$ and $\left.5^{\prime \prime}-\mathrm{H}\right), 7.15\left(1 \mathrm{H}, \mathrm{m}, 4^{\prime}-\mathrm{H}\right), 7.41\left(2 \mathrm{H}, \mathrm{d} \mathbf{J} 8.9 \mathrm{~Hz}, 2^{\prime \prime}\right.$ and 6"- $\left.\mathrm{H}\right)$, $7.56(1 \mathrm{H}, \mathrm{br} \mathrm{s}, \mathrm{NH}), 7.67\left(1 \mathrm{H}, \mathrm{dd}, \underline{\mathrm{J}}=5.1 \mathrm{and} 1.2 \mathrm{~Hz}, 5^{\prime}-\mathrm{H}\right), 7.80\left(1 \mathrm{H}, \mathrm{dd}, \underline{\mathrm{J}}=3.8\right.$ and $\left.1.2 \mathrm{~Hz}, 3^{\prime}-\mathrm{H}\right)$. MS (EI, $70 \mathrm{eV}): \mathrm{m} / \mathrm{z}$ (\%) $289\left(\mathrm{M}^{+}, 5\right), 167$ (8), 122 (9), 111 (63), 81 (14), 73 (29), 69 (71), 55 (100). IR $v_{\max }\left(\right.$ liquid film) $/ \mathrm{cm}^{-1} 1659$. 
2, 4-Dimethoxyphenyl-4-(2-thienyl)-4-oxobutanamide (4j): colorless solid mp 106-107.5 ${ }^{\circ} \mathrm{C}$ (ether). Yield 64\%. Anal. Calcd for $\mathrm{C}_{16} \mathrm{H}_{17} \mathrm{NO}_{4} \mathrm{~S}: \mathrm{C}, 60.20 ; \mathrm{H}, 5.32 ; \mathrm{N}, 4.38 ; \mathrm{S}, 10.0$. Found: C, 60.16; H, 5.39; $\mathrm{N}, 4.27 ; \mathrm{S}, 10.3 .{ }^{1} \mathrm{H}$ NMR $\delta_{\mathrm{H}}\left(\mathrm{CDCl}_{3}\right) 2.83\left(2 \mathrm{H}, \mathrm{t}, \underline{\mathrm{J}}=6.7 \mathrm{~Hz}, \underline{\mathrm{CH}}_{2}\right), 3.40\left(3 \mathrm{H}, \mathrm{t}, \underline{\mathrm{J}}=6.7 \mathrm{~Hz}, \mathrm{C}_{2}\right), 3.79$ $\left(3 \mathrm{H}, \mathrm{s}, \mathrm{OC}_{3}\right), 3.87\left(3 \mathrm{H}, \mathrm{s}, \mathrm{OC}_{3}\right), 6.51-6.55\left(2 \mathrm{H}, \mathrm{m}, 3^{\prime \prime}\right.$ and $\left.5^{\prime \prime}-\mathrm{H}\right), 7.12-7.17\left(1 \mathrm{H}, \mathrm{m}, 4^{\prime}-\mathrm{H}\right), 7.65(1 \mathrm{H}$, dd $\underline{J}=4.9$ and $\left.1.2 \mathrm{~Hz}, 5^{\prime}-\mathrm{H}\right), 7.78\left(1 \mathrm{H}, \mathrm{dd}, \underline{\mathrm{J}}=3.2\right.$ and $\left.1.2 \mathrm{~Hz}, 3^{\prime}-\mathrm{H}\right), 7.83(1 \mathrm{H}$, br s, NH), $8.20(1 \mathrm{H}, \mathrm{d}, \underline{\mathrm{J}}=$ $\left.8.6 \mathrm{~Hz}, 6^{\prime \prime}-\mathrm{H}\right)$. MS (EI, $\left.70 \mathrm{eV}\right): \mathrm{m} / \mathrm{z}(\%) 319\left(\mathrm{M}^{+}, 1\right), 256$ (3), 213 (2), 185 (2), 153 (9), 111 (6), 83 (20), 69 (67), 55 (100). IR $v_{\max }\left(\right.$ liquid film)/ $\mathrm{cm}^{-1} 1662$.

\section{General procedure for the synthesis of bithiophenes (5a-c) from the $\boldsymbol{\gamma}$-keto esters (2a-c).}

A mixture of the Lawesson's reagent $(4.8 \mathrm{~g}, 12 \mathrm{mmol})$ and the $\boldsymbol{\gamma}$-keto esters $(\mathbf{2 a - c})(10 \mathrm{mmol})$ was heated at reflux in toluene $(50 \mathrm{~mL})$ for the time indicated in Table 2. The mixture was cooled and the solvent was evaporated under reduced pressure to give the crude bithiophenes (5a-c) as oil, which were purified by "flash" chromatography on silica with increasing amounts of ether in light petroleum as eluent.

5-Methoxy-2,2'-bithiophene (5a) ${ }^{8}$ : pale yellow liquid. Yield 43\%. Anal. Calcd for $\mathrm{C}_{9} \mathrm{H}_{8} \mathrm{OS}_{2}$ : C, 55.09; H, 4.08; S, 32.68. Found: C, 55.24; H, 4.21; S, 32.70. ${ }^{1} \mathrm{H}$ NMR $\delta_{\mathrm{H}}\left(\mathrm{CDCl}_{3}\right) 3.92\left(3 \mathrm{H}, \mathrm{s}, \mathrm{OC}_{3}\right) 6.13(1 \mathrm{H}$, $\mathrm{d}, \underline{\mathrm{J}}=4.0 \mathrm{~Hz}, 4-\mathrm{H}), 6.81(1 \mathrm{H}, \mathrm{d}, \underline{\mathrm{J}}=4.0 \mathrm{~Hz}, 3-\mathrm{H}), 6.96-7.00\left(1 \mathrm{H}, \mathrm{m}, 4^{\prime}-\mathrm{H}\right), 7.02(1 \mathrm{H}, \mathrm{dd}, \underline{\mathrm{J}}=3.3$ and 1.2 $\left.\mathrm{Hz}, 3^{\prime}-\mathrm{H}\right), 7.14\left(1 \mathrm{H}, \mathrm{dd}, \underline{\mathrm{J}}=4.5\right.$ and $\left.1.2 \mathrm{~Hz}, 5^{\prime}-\mathrm{H}\right) .{ }^{13} \mathrm{C} \mathrm{NMR} \delta_{\mathrm{C}}\left(\mathrm{CDCl}_{3}\right) 60.25,104.37,121.31,122.28$, 123.22, 123.72, 127.56, 138.00, 165.48. MS (EI, $70 \mathrm{eV}): \mathrm{m} / \mathrm{z}(\%) 196\left(\mathrm{M}^{+}, 4\right), 149$ (3), 83 (10), 69 (100), 60 (82), 56 (92). IR $v_{\max }$ (liquid film)/cm-1 1564, 1523, 1487, 1450, 1424, 1353, 1320, 1252, 1228, 1198, 1151, 1079, 1052, 996, 835, 818, 771, 744, 721, 693. UV $\lambda_{\max } / \mathrm{nm}\left(\log \varepsilon_{\max } / \mathrm{dm}^{3} \mathrm{~mol}^{-1} \mathrm{~cm}^{-1}\right) 319$ (14994), 239 (4595), 207 inf. (5443).

5-Ethoxy-2,2'-bithiophene (5b) : yellow liquid. Yield 49\%. Anal. Calcd for $\mathrm{C}_{10} \mathrm{H}_{10} \mathrm{OS}_{2}$ : C, 57.13; $\mathrm{H}$, 4.76; S, 30.50. Found: C, 57.22; H, 4.91; S, 30.82. ${ }^{1} \mathrm{H} \mathrm{NMR} \delta_{\mathrm{H}}\left(\mathrm{CDCl}_{3}\right) 1.44(3 \mathrm{H}, \mathrm{t}, \underline{\mathrm{J}}=6.0 \mathrm{~Hz}$, $\left.\mathrm{OCH}_{2} \mathrm{CH}_{3}\right), 4.13\left(2 \mathrm{H}, \mathrm{q}, \underline{\mathrm{J}}=6.0 \mathrm{~Hz}, \mathrm{OC}_{2} \mathrm{CH}_{3}\right), 6.13(1 \mathrm{H}, \mathrm{d}, \underline{\mathrm{J}}=4.0 \mathrm{~Hz}, 4-\mathrm{H}), 6.80(1 \mathrm{H}, \mathrm{d}, \underline{\mathrm{J}}=4.0 \mathrm{~Hz}, 3-$ $\mathrm{H}), 6.95-6.99\left(1 \mathrm{H}, \mathrm{m}, 4^{\prime}-\mathrm{H}\right), 7.01\left(1 \mathrm{H}, \mathrm{dd}, \underline{\mathrm{J}}=3.3\right.$ and $\left.1.2 \mathrm{~Hz}, 3^{\prime}-\mathrm{H}\right), 7.13\left(1 \mathrm{H}, \mathrm{dd}, \underline{\mathrm{J}}=4.5\right.$ and $1.2 \mathrm{~Hz}, 5^{\prime}-$ $\mathrm{H}) . \delta_{\mathrm{C}}\left(\mathrm{CDCl}_{3}\right) 14.7,69.4,105.4,121.3,122.2,123.1,123.7,127.5,138.1,164.4$. MS (EI, $\left.70 \mathrm{eV}\right): \mathrm{m} / \mathrm{z}$ (\%) $210\left(\mathrm{M}^{+}, 60\right), 181$ (100), 153 (35), 109 (12), 69 (12), 45 (10). IR $v_{\max }\left(\right.$ liquid film)/cm $\mathrm{cm}^{-1}$ 1560, 1520, $1486,1469,1424,1385,1317,1249,1195,1052,878,835,766,689$. UV $\lambda_{\max } / \mathrm{nm}\left(\log \varepsilon_{\max } / \mathrm{dm}^{3} \mathrm{~mol}^{-}\right.$ $1_{\left.\mathrm{cm}^{-1}\right)} 319.5$ (14517), 240 (3892), 210 (3875).

5-Isopropyloxy-2,2'-bithiophene (5c) : yellow oil. Yield 39\%. Anal. Calcd for $\mathrm{C}_{11} \mathrm{H}_{12} \mathrm{OS}_{2}$ : C, 58.91; $\mathrm{H}$, 5.35; S, 28.60. Found: C, 59.12; H, 5.43; S, 28.55. ${ }^{1} \mathrm{H}$ NMR $\delta_{\mathrm{H}}\left(\mathrm{CDCl}_{3}\right) 1.39(6 \mathrm{H}, \mathrm{d}, \underline{\mathrm{J}}=6.1 \mathrm{~Hz}$, $\left.\mathrm{OCH}\left(\mathrm{CH}_{3}\right)_{2}\right), 4.39\left(1 \mathrm{H}, \mathrm{sep}, \underline{\mathrm{J}}=6.1 \mathrm{~Hz}, \mathrm{C} \underline{\mathrm{H}}\left(\mathrm{CH}_{3}\right)_{2}\right), 6.16(1 \mathrm{H}, \mathrm{d}, \underline{\mathrm{J}}=4.0 \mathrm{~Hz}, 4-\mathrm{H}), 6.81(1 \mathrm{H}, \mathrm{d}, \underline{\mathrm{J}}=4.0$ $\mathrm{Hz}, 3-\mathrm{H}), 6.96-6.99\left(1 \mathrm{H}, \mathrm{m}, 4^{\prime}-\mathrm{H}\right), 7.01\left(1 \mathrm{H}, \mathrm{dd}, \underline{\mathrm{J}}=4.2\right.$ and $\left.1.4 \mathrm{~Hz}, 3^{\prime}-\mathrm{H}\right), 7.13(1 \mathrm{H}, \mathrm{dd}, \underline{\mathrm{J}}=4.9$ and 1.4 $\left.\mathrm{Hz}, 5^{\prime}-\mathrm{H}\right) .{ }^{13} \mathrm{C} \mathrm{NMR} \delta_{\mathrm{C}}\left(\mathrm{CDCl}_{3}\right) 21.89,77.61,107.81,121.18,122.19,123.15,124.43,127.56,138.19$, 163.27. MS (EI, $70 \mathrm{eV}): \mathrm{m} / \mathrm{z}$ (\%) 224 (M+, 7), 182 (99), 153 (14), 111 (42), 69 (100), 58 (100), 55 (99). 
IR $v_{\max }$ (liquid film)/cm-1 2977, 1562, 1518, 1481, 1429, 1384, 1372, 1317, 1249, 1195, 1174, 1137 , 1106, 1047, 1036, 923, 823, 773, 691. UV $\lambda_{\max } / \mathrm{nm}\left(\log \varepsilon_{\max } / \mathrm{dm}^{3} \mathrm{~mol}^{-1} \mathrm{~cm}^{-1}\right) 320$ (14056), 242.5 (2944), 213 (966).

\section{General procedure for the synthesis of bithiophenes (5d-i) from the amides (4d-i).}

A mixture of the Lawesson reagent $(7.3 \mathrm{~g}, 18 \mathrm{mmol})$ and the amides (4d-i) $(18 \mathrm{mmol})$ was heated at reflux in toluene $(100 \mathrm{~mL})$ for the time indicated in Table 3. The mixture was cooled and the solvent was evaporated under reduced pressure to give the crude bithiophenes (5d-i) as oils, which were purified by "flash" chromatography on silica with increasing amounts of ether in light petroleum as eluent.

5-N,N-Dimethylamino-2,2’-bithiophene (5d) ${ }^{8}$ : yellow solid, mp 34-36 ${ }^{\circ} \mathrm{C}$. Yield. $69 \%$.

$\underline{5-N, N \text {-Diethylamino-2,2'-bithiophene (5e) }}{ }^{32}$ : yellow liquid. Yield 64\%.

5- $N, N$-Diisopropylamino-2,2'-bithiophene (5f): yellow liquid. Yield 53\%. Anal. Calcd for $\mathrm{C}_{14} \mathrm{H}_{19} \mathrm{NS}_{2}$ : $\mathrm{C}_{\text {, }}$

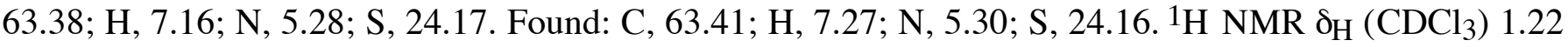
$\left(12 \mathrm{H}, \mathrm{d}, \underline{\mathrm{J}}=6.7 \mathrm{~Hz}, 4 \mathrm{xC} \underline{\mathrm{H}}_{3}\right), 3.61(2 \mathrm{H}, \mathrm{sep}, \underline{\mathrm{J}}=6.7 \mathrm{~Hz}, 2 \mathrm{xC} \underline{\mathrm{H}}), 6.15(1 \mathrm{H}, \mathrm{d}, \underline{\mathrm{J}}=3.7 \mathrm{~Hz}, 4-\mathrm{H}), 6.89(1 \mathrm{H}, \mathrm{d}$, $\underline{\mathrm{J}}=3.7 \mathrm{~Hz}, 3-\mathrm{H}), 6.96\left(1 \mathrm{H}, \mathrm{m}, 4^{\prime}-\mathrm{H}\right), 7.00\left(1 \mathrm{H}, \mathrm{dd}, \underline{\mathrm{J}}=3.7\right.$ and $\left.1.2 \mathrm{~Hz}, 3^{\prime}-\mathrm{H}\right), 7.09(1 \mathrm{H}, \mathrm{dd}, \underline{\mathrm{J}}=4.8$ and 1.2 $\left.\mathrm{Hz}, 5^{\prime}-\mathrm{H}\right) .{ }^{13} \mathrm{C} \mathrm{NMR} \delta_{\mathrm{C}}\left(\mathrm{CDCl}_{3}\right) 20.87,50.88,113.44,121.40,121.96,122.39,125.53,127.50,138.82$, 153.10. MS (EI, $70 \mathrm{eV}): \mathrm{m} / \mathrm{z}$ (\%) 265 (M+, 54), 250 (16), 222 (27), 208 (26), 180 (54), 69 (100). IR $v_{\max }\left(\right.$ liquid film)/cm ${ }^{-1}$ 3070, 2970, 2930, 2870, 1557, 1511, 1489, 1380, 1366, 1331, 1316, 1271, 1247, $1230,1190,1157,1125,1055,1014,920,885,839,798,757,688$. UV $\lambda_{\max } / \mathrm{nm}\left(\log \varepsilon_{\mathrm{max}} / \mathrm{dm}^{3} \mathrm{~mol}^{-1} \mathrm{~cm}^{-}\right.$ 1) 341.0 (11494), 246.5 (4481), 213.5 (3633).

5-Piperidino-2,2'-bithiophene (5g): pale yellow solid, mp 59-60 ${ }^{\circ} \mathrm{C}$ (light petroleum). Yield 79\%. Anal. Calcd for $\mathrm{C}_{13} \mathrm{H}_{15} \mathrm{NS}_{2}$ : C, 62.64; H, 6.02; N, 5.62; S, 25.73. Found: C, 62.59; H, 6.09; N, 5.56; S, 26.03. ${ }^{1} \mathrm{H}$ NMR $\delta_{\mathrm{H}}\left(\mathrm{CDCl}_{3}\right) 1.50-1.64\left(2 \mathrm{H}, \mathrm{m}, \mathrm{CH}_{2}\right), 1.68-1.80\left(4 \mathrm{H}, \mathrm{m}, 2 \mathrm{xCH}_{2}\right), 3.10-3.20(4 \mathrm{H}, \mathrm{m}$, $\left.2 \mathrm{xC} \underline{\mathrm{H}}_{2}\right), 6.00(1 \mathrm{H}, \mathrm{d}, \underline{\mathrm{J}}=4.0 \mathrm{~Hz}, 4-\mathrm{H}), 6.89(1 \mathrm{H}, \mathrm{d}, \underline{\mathrm{J}}=4.0 \mathrm{~Hz}, 3-\mathrm{H}), 6.95-7.00\left(2 \mathrm{H}, \mathrm{m}, 3^{\prime}\right.$ and $\left.^{\prime}-\mathrm{H}\right)$, 7.05-7.11 (1H, m, 5'-H). ${ }^{13} \mathrm{C} \mathrm{NMR} \delta_{\mathrm{C}}\left(\mathrm{CDCl}_{3}\right)$ 23.60, 25.09, 52.58, 105.48, 121.39, 122.43, 123,04, 127.51, 138.48, 158.44. MS (EI, $70 \mathrm{eV}): \mathrm{m} / \mathrm{z}(\%) 249\left(\mathrm{M}^{+}, 100\right), 69$ (9), 57 (14). IR $v_{\max }($ liquid film)/cm $\mathrm{cm}^{-1} 2939,2826,1556,1514,1488,1446,1301,1276,1242,1192,1121,1068,1013,894,826$, 810, 769, 682. UV $\lambda_{\max } / \mathrm{nm}\left(\log \varepsilon_{\max } / \mathrm{dm}^{3} \mathrm{~mol}^{-1} \mathrm{~cm}^{-1}\right) 343.0$ (18039), 241.5 (4914), 214.0 (6102).

5- $N, N$-Dicyclohexylamino-2,2'-bithiophene (5h): pale yellow solid, mp 52-55 ${ }^{\circ} \mathrm{C}$ (light petroleum). Yield 63\%. ${ }^{1} \mathrm{H} \mathrm{NMR} \delta_{\mathrm{H}}\left(\mathrm{CDCl}_{3}\right)$ 0.90-1.82 $\left(20 \mathrm{H}, \mathrm{m}, 10 \mathrm{xC} \underline{H}_{2}\right), 3.00-3.20(2 \mathrm{H}, \mathrm{m}, \mathrm{C} \underline{\mathrm{H}}), 6.19(1 \mathrm{H}, \mathrm{d}, \underline{\mathrm{J}}=3.7 \mathrm{~Hz}$, 4-H), $6.86(1 \mathrm{H}, \mathrm{d}, \underline{\mathrm{J}}=3.7 \mathrm{~Hz}, 3-\mathrm{H}), 6.90-7.25\left(2 \mathrm{H}, \mathrm{m}, 3^{\prime}\right.$ and $\left.4^{\prime}-\mathrm{H}\right), 7.04$ (1H, br d, $\left.\underline{\mathrm{J}}=5.2 \mathrm{~Hz}, 5^{\prime}-\mathrm{H}\right) . \mathrm{MS}$ (EI, 70 eV): m/z (\%) 345 (M+, 100), 302 (4), 263 (31), 236 (5), 220 (9), 205 (8), 192 (5), 180 (57), 153, (5), 121 (8), 96 (14), 81 (11), 55 (30). IR $v_{\max }($ liquid film)/cm-1 2940, 2860, 1520, 1480, 1450, 1380, 1340, 1260, 1240, 1200, 1130, 1120, 1110, 1050, 1020, 980, 900, 840, 800, 760, 715, 690. 
5-(4-Methoxyanilino)-2,2'-bithiophene (5i): pale green solid, mp 113-115 ${ }^{\circ} \mathrm{C}$ (ether). Yield 14\%. Anal. Calcd for $\mathrm{C}_{15} \mathrm{H}_{13} \mathrm{NOS}_{2}$ : C, 62.71; H, 4.52; N, 4.87; S, 22.32. Found: C, 62.84; H, 4.61; N, 4.81; S, 22.72. ${ }^{1} \mathrm{H} \mathrm{NMR} \delta_{\mathrm{H}}\left(\mathrm{CDCl}_{3}\right) 3.80\left(3 \mathrm{H}, \mathrm{s}, \mathrm{OC}_{3}\right), 6.50(1 \mathrm{H}, \mathrm{d}, \underline{\mathrm{J}}=3.7 \mathrm{~Hz}, 4-\mathrm{H}), 6.85(2 \mathrm{H}, \mathrm{d}, \underline{\mathrm{J}}=8.9 \mathrm{~Hz}$, $2^{\prime \prime}$ and $6^{\prime \prime}-\mathrm{H}$ or $3^{\prime \prime}$ and $\left.5^{\prime \prime}-\mathrm{H}\right), 6.94(1 \mathrm{H}, \mathrm{d}, \underline{\mathrm{J}}=3.7 \mathrm{~Hz}, 3-\mathrm{H}), 6.97\left(2 \mathrm{H}, \mathrm{d}, \underline{\mathrm{J}}=8.9 \mathrm{~Hz}, 3^{\prime \prime}\right.$ and 5"'-H or $2^{\prime \prime}$ and $\left.\quad 6^{\prime \prime}-\mathrm{H}\right), 7.00\left(1 \mathrm{H}, \mathrm{m}, 4^{\prime}-\mathrm{H}\right), 7.05\left(1 \mathrm{H}, \mathrm{dd}, \underline{\mathrm{J}}=3.6\right.$ and $\left.1.2 \mathrm{~Hz}, 3^{\prime}-\mathrm{H}\right), 7.16(1 \mathrm{H}, \mathrm{dd}, \underline{\mathrm{J}}=5.0$ and $\left.1.2 \mathrm{~Hz}, 5^{\prime}-\mathrm{H}\right) .{ }^{13} \mathrm{C} \mathrm{NMR} \delta_{\mathrm{C}}\left(\mathrm{CDCl}_{3}\right) 55.62,114.74,115.49,117.54,122.35,122.45,123.34,127.63$, 127.92, 137.99, 138.36, 147.29, 154.27. MS (EI, $70 \mathrm{eV}): \mathrm{m} / \mathrm{z}$ (\%) 287 (M+, 100), 272 (70), 210 (9), 185 (11), 121, (13), 96 (14), 77 (6), 63 (7). IR $v_{\max }($ liquid film)/cm-1 1514, 1462, 1441, 1349, 1298, 1249, 1165, 1102, 1040, 916, 835, 789. UV $\lambda_{\max } / \mathrm{nm}\left(\log \varepsilon_{\max } / \mathrm{dm}^{3} \mathrm{~mol}^{-1} \mathrm{~cm}^{-1}\right) 365.5$ (19900), 245.0 (10596), 203.5 (31029).

\section{ACKNOWLEDGEMENTS}

Thanks are due to FCT through IBQF (UM), ICCTI/French Embassy (Technical and Scientific Cooperation Programme) and to Dra. A.M.F. Oliveira-Campos for the interesting discussions.

\section{REFERENCES}

1. P. N. Prasad and D. J. Williams, Introduction to non-linear effects in molecules and polymers, New York, Wiley, 1991.

2. D. S. Zyss, Non-linear optical properties of organic molecules and crystals, Vol. 1 and 2; Orlando, Academic Press, 1987.

3. C. Brosshard, K. Sutter, P. Petre, J. Hulliger, M. Florsheimer, M. Kaatz, and P. Gunter, Organic nonlinear optical materials, Amsterdam, Gordon and Breach Science Publishers, 1995.

4. G. Mignani, F. Leising, R. Meyrueix, and M. Samson, Tetrahedron Lett., 1990, 31, 4743.

5. M. G. Hutchins, I. Ferguson, D. J. McGeein, J. O. Morley, J. Ziss, and I. Ledoux, J. Chem. Soc., Perkin Trans. 2, 1995, 175.

6. F. Wurthner and F. Effenberg, Chem. Phys., 1993, 173, 305.

7. F. Effenberger and F. Wurthner, Angew. Chem., Int. Ed. Eng., 1993, 32, 719.

8. F. Effenberger, F. Wurthner, and F. Steybe, J. Org. Chem., 1995, 60, 2082.

9. C. Branger, M. Lequan, R.M. Lequan, M. Barzoukas, and A. Fort, J. Mater. Chem., 1996, 6, 555.

10. F. Steybe, F. Effenberger, S. Beckman, P. Kramer, C. Glania, and R. Wortmann, Chem. Phys., 1997, 219, 317.

11. F. Steybe, F. Effenberger, S. Beckman, U. Gluber, C. Bosshard, and P. Gunter, Tetrahedron, 1998, 54, 8469.

12. H. S. Vollmer, F. Effenberger, T. Stumpfig, A. Hartschuh, H. Port, and H. C. Wolf, J. Org. Chem., 1998, 63, 5080.

13. F. Effenberger and G. Grube, Synthesis, 1998, 1372. 
14. M. S. Vollmer, F. Wurthner, F. Effenberger, P. Emele, D. V. Meyer, T. Stumpfig, P. Port, and H.C. Wolf, Chem. Eur. J., 1998, 4 , 260.

15. J. M. Tour and R. Wu, Macromolecules, 1992, 25, 1901.

16. G. Barbarella, A. Bongini, and M. Zambianchi, Macromolecules, 1994, 27, 3039.

17. G. Barbarella and M. Zambianchi, Tetrahedron, 1994, 50, 11249.

18. U. Folli, D. Iarossi, M. Montorsi, A. Mucci, and L. Schenetti, J. Chem. Soc., Perkin Trans. 1, 1995, 537.

19. R. Shabana, A. Galal, H. B. Mark, H. Zimmer, S. Gronowitz, and A. B. Hoernfeldt, Phosphorus Sulfur, 1990, 48, 239.

20. A. Amer, A. Burkhardt, A. Nkansah, R. Shabana, A. Galal, H. B. Mark, and H. Zimmer, Phosphorus Sulfur, 1989, 42, 63.

21. T. Sone, Y. Umetsu, and K. Sato, Bull. Chem. Soc. Jpn., 1991, 64, 864.

22. T. Sone, K. Sato, Y. Umetsu, A. Yoshimo, and K. Takahashi, Bull. Chem. Soc. Jpn., 1994, 67, 2187.

23. F. Freeman, H. Lu, Q. Zeng, and E. Rodriguez, J. Org. Chem., 1994, 59, 3665.

24. T. Masquelin and D. Obrecht, Tetrahedron Lett., 1994, 35, 9387.

25. D. Prim and G. Kirsch, J. Chem. Soc., Perkin Trans. 1, 1994, 2603.

26. D. Prim, G. Kirsch, F. Leising, and G. Mignani, J. Heterocycl. Chem., 1994, 31, 1005.

27. Dictionary of Organic Compounds; 1965, Vol. 5, ed. by J. R. Pollock and R. Stevens, Eyre and Spottiswoode Ltd and E. F. N. Spon Ltd Publishers, London.

28. B. P. Fabrichnyi, I. F. Shalavina, and Ya. L. Gol’dfarb, Zhur. Obshchei Kim., 1958, 28, 213 (Chem. Abstr., 1959, 53, 3052f).

29. H. Stetter, M. Schreckenberg and K. Wiemann, Chem. Ber., 1976, 109, 541.

30. L. Fieser and R.G. Kennelly, J. Am. Chem. Soc., 1935, 57, 1616.

31. T. Nishio, Helv. Chim. Acta, 1998, 81, 1207.

32. R. L. P. De Long and L. Brandsma, J. Organomet. Chem., 1986, 316, C21-C23. 